\title{
Periarticular Bone Changes in Osteoarthritis
}

\author{
Harrie Weinans, PhD
}

Received: 31 August 2011/Accepted: 29 November 2011/Published online: 24 January 2012

(C) The Author(s) 2012. This article is published with open access at Springerlink.com

Keywords osteoarthritis $\cdot$ subchondral bone - osteoclast . micro-SPECT·micro-CT

\section{Introduction}

Osteoarthritis (OA) can be considered an organ failure with pathological aspects in cartilage, bone, ligaments, and synovium. Altogether, these tissue changes can result in pain and immobilization - a failure of the joint. It is well regarded that $\mathrm{OA}$ is a complex multifactorial disease with many risk factors and different etiological pathways that all lead to an apparently similar end stage. Bony changes are clearly observed in advanced OA. However, little understanding exists on the role of these changes, whether they are a consequence of cartilage damage or precede this damage and maybe play an important role in the etiological process. Even more important is the issue of pain. Radiological scores of OA do not match well with pain and mobility scores, which questions the value of these scoring systems. It seems that we do not assess the most relevant parameters. Evaluation of conventional and new radiographic parameters is still an extensive part of the OA research field. We may have overlooked certain (subtle) parameters that can be extracted from x-rays, but other imaging modalities such as MRI, CT, or SPECT might better represent $\mathrm{OA}$ in a clinically relevant manner.

H. Weinans $\operatorname{PhD}(\bowtie)$

Orthopaedic Research Laboratory,

Erasmus MC,

Room EE-1614, P.O. Box 2040,

3000 CA Rotterdam, The Netherlands

e-mail: h.weinans@erasmusmc.nl

\section{Subchondral Bone Adaptation in Osteoarthritis}

Radin and Rose [9] postulated that local subchondral bone stiffening generates steep stiffness gradients and thereby induces high shear forces in the cartilage that subsequently leads to deterioration. Hence, osteoarthritis might begin with subchondral bone adaptation and stiffness gradients. However, this hypothesis was difficult to prove and was never supported by experimental data. Later, Li and Aspden [4] and Day et al. [2] showed that the stiffness of the high-density subchondral bone in OA is not as high as expected from its density (i.e., its volume fraction). The high density is possibly a compensation for the low mineralization caused by the high turnover rate in this area (Fig. 1). Apparently, the gradients in stiffness are less than assumed from the gradients in density.

Another interesting hypothesis came from the work of Westacott et al. [13], who found that bone cells from OA patients can adversely influence cartilage metabolism. This provided a hint that the increased subchondral bone activity in OA joints can lead to cartilage degeneration. Of course, this hypothesis requires diffusion of harmful agents between the subchondral bone compartment and the cartilage, which might be difficult if the mineralized cartilage and subchondral plate are relatively dense. To further investigate the role of subchondral bone in OA, we decided to first investigate the cascade of events in subchondral bone during the progression of OA in animal models using micro-CT for in vivo evaluations at various time points after induction of OA.

\section{Observations from a Murine Model of Osteoarthritis}

In the knee of mice, OA was induced by bacterial type VII collagenase injections. This model results in ligament rather than cartilage degeneration [11] and can be considered a chemical ligament resection leading to joint instability. The consequences for the subchondral bone plate are thinning (from about 140 to $110 \mu \mathrm{m}$ ) at short 


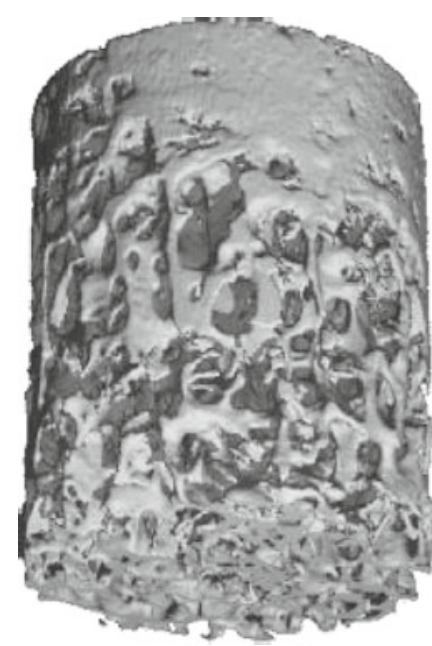

$\mathrm{OA}$

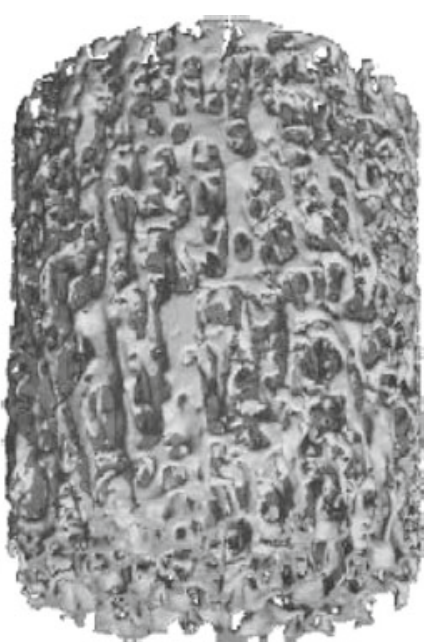

healthy

Fig. 1. Representative sample from human proximal tibia with mild $\mathrm{OA}$ vs. sample from healthy control. Although the density in the OA sample is clearly higher, the mechanical stiffness is almost the same. Analyses from Day et al. [2] showed that at the tissue level the elastic modulus in mild OA subchondral bone is about $50 \%$ less than in healthy tissue. As a consequence, the overall stiffness in the higher density OA bone is about the same as the healthy tissue

term (2 weeks) with later thickening. In this model, subchondral plate perforations as a consequence of increased osteoclast activity could be determined as well, and from 2 weeks on, the number of perforations through the subchondral plate and calcified cartilage was markedly increased (Fig. 2) [1]. Cartilage damage and osteophytes also develop in this model.

Evaluation of bone with in vivo micro-CT is an established method [12], but cartilage visualization can be performed as well using the contrast agent Hexabrix (Fig. 3) [7]. The latter enables visualization of cartilage glycosaminoglycan (GAG) concentration, similar to assessment by dGEMRIC in humans. Various animal models have a different time frame of OA progression and localization of cartilage degeneration and bone erosion [10].
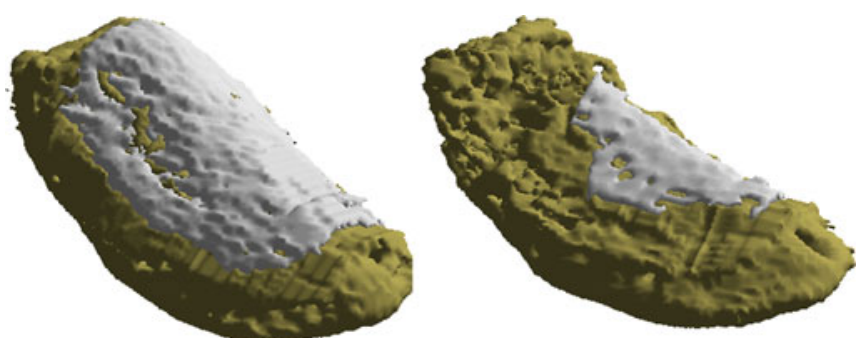

Fig. 3. Cartilage degeneration in rat monoiodoacetate (MIA) model 4 days after MIA injection (left) and 44 days after injection. At 4 days, the cartilage was virtually intact, whereas after 44 days, cartilage loss was considerable with severe subchondral erosion

We attempted to image the higher osteoclast activity (or bone turnover rate) that generates the bone erosion by other more direct means. In a rat model using monoiodoacetate (MIA) to induce OA, a bone scan was made with a novel multi-pinhole SPECT. Methyl diphosphonate labeled with $99 \mathrm{~m}$-technetium was used $\left({ }^{99 \mathrm{~m}} \mathrm{Tc}-\mathrm{MDP}\right)$ as a radioactive tracer. As early as 2 days after induction of OA, the SPECT scan showed higher signal intensity (Fig. 4), indicating increased osteoclast activity. Since the growth plate in the rat is not closed, the overall signal intensity around the knee is high. However, the high resolution animal SPECT is able to differentiate the signals from growth plate and subchondral bone. Histology of the same knees 2 days later (i.e., 4 days after MIA injections) could not distinguish the cartilage from the treated knees and the healthy contralateral control knees. At later time points (16 and 44 days after the injections), a clear difference was seen with lower GAG staining, fibrillation, and chondrocyte clustering.

Surprisingly, higher bone turnover is an extremely fast response in the MIA model, contrary to the idea that MIA directly targets the cartilage, thereby inducing OA. This finding could be interpreted as the bone alteration occurring first. However, care must be taken with this interpretation. What we call "first" also relates to how much we can see with our imaging and/or
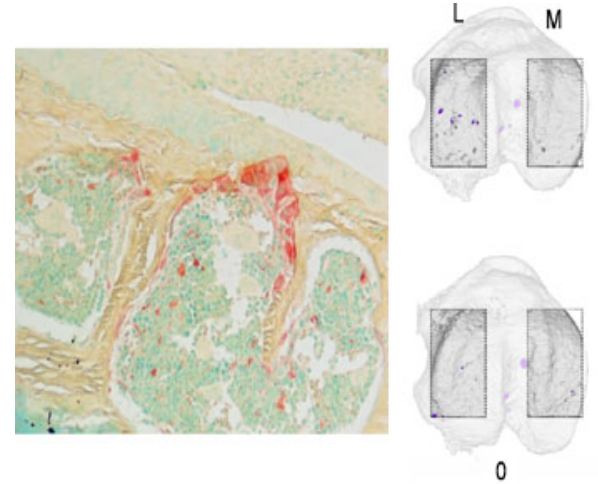

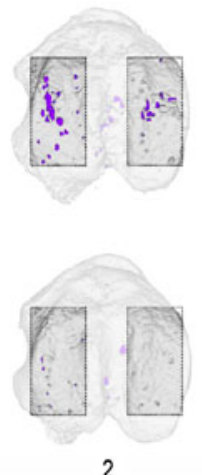

2
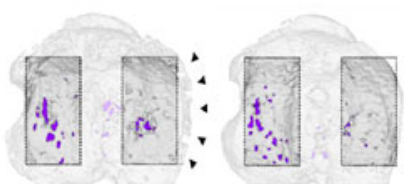

OA-induced side

$$
\text { weeks post injection }
$$

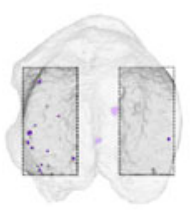

4

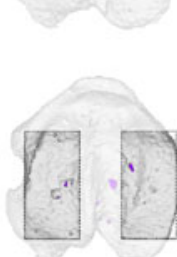

14

Fig. 2. Perforations of the subchondral plate and calcified cartilage through increased osteoclast activity. Histology with specific osteoclast staining (left) and counted perforations at different time points measured with in vivo micro-CT (right) 

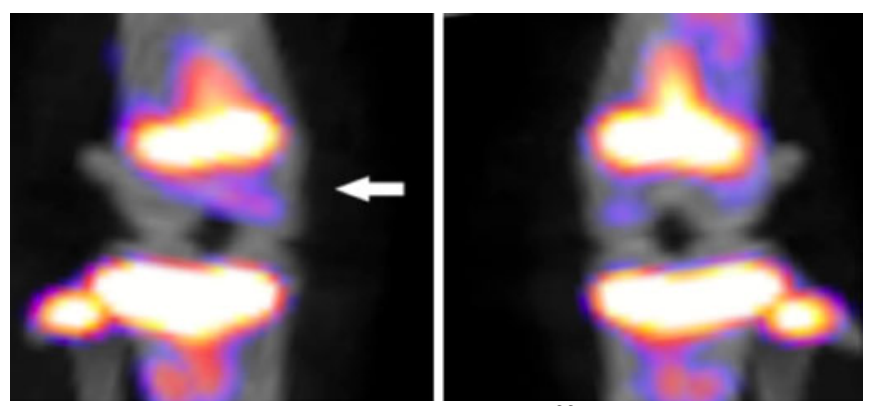

Fig. 4. Multi pinhole SPECT scan using ${ }^{99 \mathrm{~m}}$ Tc-MDP to visualize bone turnover in MIA-induced OA rat model. At the left, the OA side vs. the contralateral control side at the right. There was a higher signal in the subchondral bone (white arrow) already 2 days after OA induction. The other large signal is from growth plate

histological methods. The bone SPECT scan is an extremely sensitive tool for measuring a bone response, and the cartilage might have been responsive to the MIA trigger at the 2-day time point as well, but maybe the histology was not sensitive enough to show it.

\section{Summary}

The animal models and the imaging tools provide a powerful combination for studying OA. What did we learn from the current approaches? It has been known for some years that subchondral bone is a highly adaptive tissue, and changes in subchondral bone can be accurately visualized and quantified by CT [6] and scintigraphy [5] even in a clinical setting. Animal CT and SPECT have been improved in the last decade to higher resolutions [3, 12], enabling refined in vivo evaluation in small animal research. Besides evaluations of subchondral bone and cartilage with the microimaging modalities, SPECT/CT can image macrophage activation as well [8]. With repetitive imaging of animals, we will learn the precise sequence of events that occur in the various tissues of the diseased joint. The bone scan analyses, with its fast (2 days) response in bone turnover in the MIA OA model, substantiate the often suggested crosstalk between the bone and cartilage compartments. In the different animal models, this sequence of events might be different; in one model, the problems might start in the cartilage, whereas in another model the bone might be triggered first. Each model might expose a different etiological pathway, similar to the differences in OA etiology in humans. Hopefully, these new tools will elucidate concepts and targets that help us in the design of new therapeutic interventions for $\mathrm{OA}$.
Disclosures The author certifies that he has no commercial associations (e.g., consultancies, stock ownership, equity interest, patent/licensing arrangements, etc.) that might pose a conflict of interest in connection with the submitted article.

The author certifies that his institution has approved the animal protocol for this investigation and that all investigations were conducted in conformity with ethical principles of research.

Open Access This article is distributed under the terms of the Creative Commons Attribution Noncommercial License which permits any noncommercial use, distribution, and reproduction in any medium, provided the original author(s) and source are credited.

\section{References}

1. Botter SM, van Osch GJ, Clockaerts S, Waarsing JH, Weinans $\mathrm{H}$, van Leeuwen JP. Osteoarthritis induction leads to early and temporal subchondral plate porosity in the tibial plateau of mice: An in vivo micro CT study. Arthritis Rheum. 63:2690-9, 2011

2. Day JS, Ding M, van der Linden JC, Hvid I, Sumner DR, Weinans H. A decreased subchondral trabecular bone tissue elastic modulus is associated with pre-arthritic cartilage damage. J Orthop Res 19:914-8, 2001.

3. van der Have F, Vastenhouw B, Ramakers RM, Branderhorst W, Krah JO, Ji C, Staelens SG, Beekman FJ. U-SPECT-II: An Ultra-High-Resolution Device for Molecular Small-Animal Imaging. J Nucl Med 50:599-605, 2009

4. Li B, Aspden RM. Mechanical and material properties of the subchondral bone plate from the femoral head of patients with osteoarthritis or osteoporosis. Ann Rheum Dis 56:247-54, 1997

5. McCrae F, Shouls J, Dieppe P, Watt I. Scintigraphic assessment of osteoarthritis of the knee joint. Ann Rheum Dis 51:938-42, 1992.

6. Müller-Gerbl M. The subchondral bone plate. Adv Anat Embryol Cell Biol 141:III-XI, 1-134, 1998.

7. Piscaer TM, Waarsing JH, Kops N, Pavljasevic P, Verhaar JA, van Osch GJ, Weinans $\mathrm{H}$. In vivo imaging of cartilage degeneration using microCT-arthrography. Osteoarthritis Cartilage 16:1011-7, 2008

8. Piscaer TM, Müller C, Mindt TL, Lubberts E, Verhaar JA, Krenning EP, Schibli R, De Jong M, Weinans H. Imaging of activated macrophages in experimental osteoarthritis using folatetargeted animal single-photon-emission computed tomography/ computed tomography. Arthritis Rheum 63:1898-907, 2011

9. Radin EL, Rose RM. Role of subchondral bone in the initiation and progression of cartilage damage. Clin Orthop Relat Res 213:34-40,1986

10. Siebelt M, Waarsing JH, Kops N, Piscaer TM, Verhaar JA, Oei $\mathrm{EH}$, Weinans H. Quantifying osteoarthritic cartilage changes accurately using in vivo microCT arthrography in three etiologically distinct rat models. J Orthop Res. 29:1788-94, 2011.

11. van Osch GJ, van der Kraan PM, Blankevoort L, Huiskes R, van den Berg WB. Relation of ligament damage with site specific cartilage loss and osteophyte formation in collagenase induced osteoarthritis in mice. J Rheumatol 23:1227-32, 1996

12. Waarsing JH, Day JS, van der Linden JC, Ederveen AG, Spanjers C, De Clerck N, Sasov A, Verhaar JA, Weinans H. Detecting and tracking local changes in the tibiae of individual rats: a novel method to analyse longitudinal in vivo micro-CT data. Bone 34:163-9, 2004.

13. Westacott CI, Webb GR, Warnock MG, Sims JV, Elson CJ. Alteration of cartilage metabolism by cells from osteoarthritic bone Arthritis Rheum 40:1282-91, 1997 